$\square$ $\square$

$\nabla$

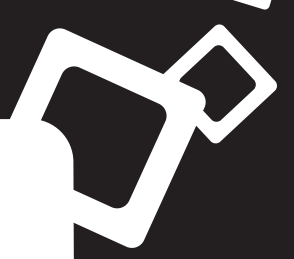

DOCUMENTOS INSTITUCIONALES

* Apuesta estratégica para el desarrollo futuro del Centro de Investigación Científica CINC-ANSP 
$\square$

$\square$

$\triangle$

* Project for the strategic transformation of the Scientific Research Center. 
MEMORANDO MCINC50 09-2013

Para: Director General ANSP

c.c.: Subdirector Ejecutivo ANSP

De: Coordinador Principal CINC-ANSP

Asunto: Apuesta estratégica para el desarrollo futuro del CINC

Fecha: 30 de octubre de 2013

Por este medio hago entrega de documento estratégico intitulado: "Apuesta estratégica para el desarrollo futuro del Centro de Investigación Científica CINCANSP"; en el cual encontrará una descripción de los desafíos que enfrentará esta unidad en los años venideros. Esta elaborado en un espiritu de aporte hacia la gran estrategia de esta institución en materia de investigación científica.

La concepción estratégica instalada transversalmente en el documento contribuirá con insumos fundamentales al diseño de agendas institucionales e incluso planes quinquenales. Algunos de sus contenidos son para su consideración, otros para identificar los puntos de apoyo necesarios para crecer como experiencia exitosa de la actual administración, en cuanto uno de sus principales productos incluye una revista élite indizada internacionalmente; siendo la primera del sector público. Además de contar con todo un programa de investigación científica que supera con creces lo planteado por el Estado mismo en el rubro seguridad.

Para su conocimiento y reflexión.

Sin otro particular

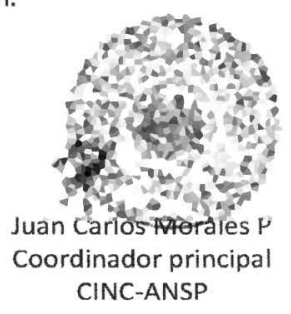



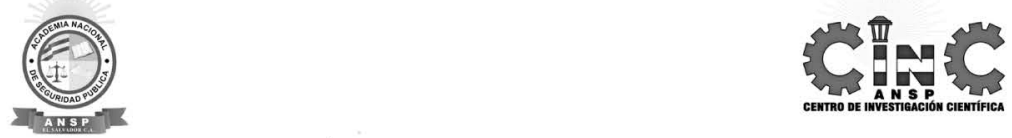

\section{Apuesta estratégica para el desarrollo futuro del Centro de Investigación Científica CINC-ANSP (Documento estratégico)}

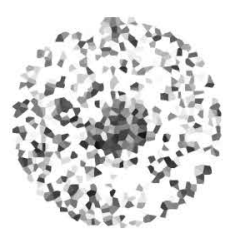



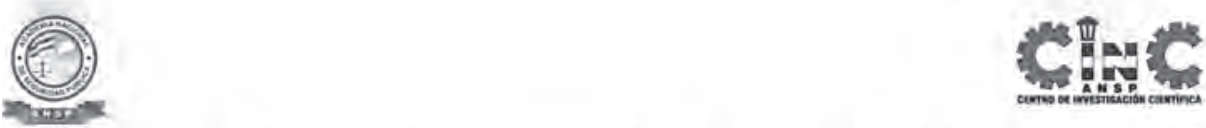

Por este medio me permito exponer asuntos relacionados entre sí a manera de apuestas estratégicas para el desarrollo futuro del Centro de Investigación Cientifica.

En mi calidad de Coordinador Principal expreso que ha sido mi filosofia de trabajo, la de aportar creatividad e innovación en todos nuestros empenos; haciéndolo no sólo para un fin administrativo que se refiere al cumplimiento de metas; sino más importante aún para dotar a la institución y el pais de un producto vanguardista de mucha calidad y que es necesario como insumo vital para la atención de los problemas de seguridad.

En la vision del Centro de Investigación Cientifica y que reza asi: "Ser un ente de alto prestigio nacional e internacional de referencia centroamericana reconocido por su aporte al fortalecimiento institucional, el diseño inteligente de las políticas de seguridad pública y la innooación en los procesos educatioos intervinientes en la formación policial". De este se desprenden tres ejes estratégicos en los que estamos decididos a impactar.

\section{Sobre fortalecimiento institucional}

En cuanto a este factor, hemos logrado posicionar estratégicamente una revista académica, "Policia y Seguridad Pública" que tiene el formato de un journal científico, indizado en bases de datos internacionales (Latindex, Lamjol y Google Académico); que repercutirá positiva e inevitablemente en dos aspectos fundamentales:

1. En primer lugar, llenará un vacio en materia de investigación científica en el nicho del sector público, y en la materia específica de seguridad. Dotando en el proceso a decisores, planificadores, asesores y demás élites de la burocracia gubernamental de herramientas vatiosas y planteamientos innovadores.Posibilitando sinergias interesantes tipo asocios estratégicos, que han estado gestándose y desarrollándose con entidades tales como: Dirección General de Centros Penales (DGCP); Instituto Nacional de la Salud (INS); Centro Nacional de Investigaciones en Ciencias Sociales y Humanidades (CENISCH); Colegio de Altos Estudios Estratégicos (CAEE); la Unidad de Análisis y Monitoreo del Ministerío de Justicia y Seguridad Pública, entre otras.

2. En segundo lugar, contribuirá substancialmente en la habilitación y permanencia de la función estratégica referida a investigación cientifica, sobre todo porque el Centro de Investigación Científica ha propiciado estudios e investigaciones de tipo politico - estratégica, es decir aquella que sirve en los niveles decisores de la alta política; y porque las temáticas tratadas derivan de una agenda institucional autorizada que se relaciona 
con los programas de investigación cientifica que se van activando progresivamente. Lo anterior indudablemente será de apoyo fundamental al proceso de potenciación de la ANSP como Instituto de Educación Superior (IES).

En cuanto a la generación de propuestas avanzadas, aspiramos a impactar decisivamente en el diseño, formulación y evaluación de políticas públicas de seguridad; deseamos que la aún incipiente plataforma institucional del CINC se convierta en un embrión de ente especializado con una oferta de producción científica consistente y apoyada en una convocatoria amplia de especialistas y profesionales tanto nacionales como internacionales. Para ello una misión de rigor será la propiciación de la incidencia social y la implicación estratégica, para lo cual los productos, entendiéndose los estudios e investigaciones deberán tener las siguientes caracteristicas:

1. Atender los programas de investigación vigentes o activos;

2. Los programas de investigación deben estar relacionados estratégicamente con los ejes referidos a:

a) prevención de violencia y convivencia ciudadana;

b) estudios sobre el futuro y gestion de entornos complejos de seguridad;

c) diseño inteligente de politicas públicas;

d) recursos humanos policiales e

e) innovación educativa policial;

3. Deben ser estudios y aportes académicos novedosos, innovadores, inéditos y sin publicaciones previas;

4. Las investigaciones deben adoptar la fisonomía de artículos académicos / científicos, estudios estratégicos o balances estratégicos que constituyen los formatos declarados en la publicación y

5. Deben contener componentes de uso potencial para quienes se dirija, implicando aspectos prácticos y vanguardistas.

Para todo lo anterior el equipo CINC en su rol de liderazgo de la gestión editorial fortalecerá los arbitrajes, potenciará los contactos para estudios adhoc, acompañará a los autores en el desarrollo de sus estudios y propondrá fichas de estudio para su designación entre los investigadores de la comunidad académica. 

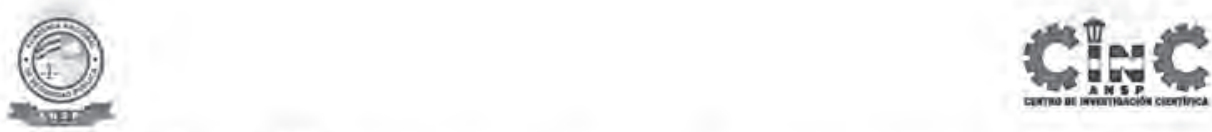

Sobre políticas públicas

En cuanto a estas necesitamos pensar distinto, reaprender de todo aquello que creímos entender, planteando la profesionalizacion del asesoramiento de alto nivel, habilitando intersecciones entre instituciones públicas a fin de garantizar confecciones valiosas de políticas. Uno de los primeros trabajos publicados en la revista "Policia y Seguridad Pública" especificamente uno referido al municipio de Santa Tecla; impactó de tal forma la estrategia de reforma urbana de la Alcaldia Municipal de Santa Tecla; implicando un alto hasta lo que entonces se había hecho en la recuperación de espacios públicos, el concepto de Paseo El Carmen fue extendido más allá del circuito geográfico originalmente concebido para adentrarse a la zona norponiente, la más deprimida históricamente de este municipio. En el proceso revaluó propiedades, trajo consigo nuevos emprendedurismos y concedió a sus habitantes pobres la posibilidad de acceder a un mayor umbral de habitabilidad urbana. El estudio incidió en la remoción definitiva de promotorios ilegales de basura, redefinió la reubicación de talleres y otras infraestructuras que servían de cobertura al contrabando y generaban desorden territorial y aseguró una ruta peatonal y ciclovía que une a la ciudad de poniente a oriente (yendo hacia El Cafetalón). Lo anterior confirmará una nueva ecuación de convivencia y disipará los temores hacia zonas anteriormente periféricas.

Así también el estudio, siguió impactando al quehacer de otras instituciones, como por ejemplo el Ministerio de Obras Públicas (MOP); que comprendió que una vía de comunicación como el ex Boulevard Diego de Holguín no podia conectar con la carretera Panamericana que conduce hacia occidente, por ser esta última una vía de acceso muy deficiente e incapaz de descomplicarel flujo vehicular de la primera; el estudio lo planteaba y fue tomando en cuenta para la modernización de la ruta.

Estas líneas describen la lógica y nivel de incidencia de cómo los estudios e investigaciones deben impactarlos esfuerzos estatales, por ello se escogen temáticas vitales y se validan con la necesidades sociales y de Estado.

Sobre innovación educativa

En un tercer aspecto referido a la innovación en la educación policial, la perspectiva y la visión es sobrepasar conceptos tradicionales, es decir aquellos estudios centrados en plantillas policiales cuya lógica cuantitativa guarda distancia de la necesidad de conocer los entornos laborales y de trabajo en los que se desenvuelven; reforzando como algo relevante el examen minucioso de la oferta educativa, haciendo el escrutinio debido de las herramientas adecuadas con las que se forman para la gestión de la realidad de seguridad. El valor agregado de estas investigaciones escudrina en aristas de análisis poco exploradas, en la propuesta de rutas vanguardistas, en definitiva en la 

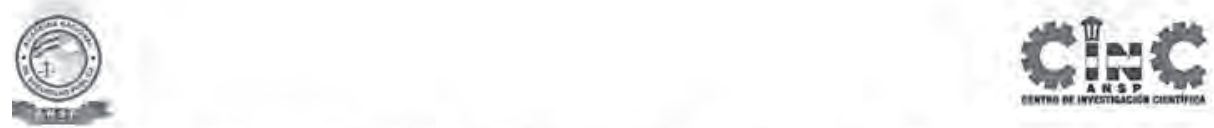

transformación genuina y real de la educación policial, apoyándose para ello en la introducción de disciplinas científicas diversas, que permitan la sólida lectura de los problemas desde la sociologia de las relaciones internacionales para entender dinámicas transnacionales; la psicologia para comprender incentivos morales y estados de ánimo; la ciencia política para el estudio de las relaciones de poder y la lógica administrativa; la medicina para expanderse a la epidemiología y los estados de salud de segmentos de población específica.

Para continuar con esta concepción estratégica de trabajo, el Centro de Investigación Cientifica deberá enfrentar desafios importantes; de cuya inteligente gestión dependerá el aporte que la ANSP haga en materia de investigación científica para la seguridad y por lo tanto atendiendo uno de los problemas fundamentales del país.

\section{Desafio estratégico \# 1}

La ANSP deberá avanzar hacia el diseño, formulación e implementación de una política de investigación científica institucional. Es una meta que puede ser asequible en un quinquenio y que debe contar con distintos componentes claves. Uno de ellos se refiere al concepto de producción cientifica que debe habilitar y desarrollar un tipo de investigación educativo-curricular e institucional y por otra parte la consolidación y potenciación de otra investigación de indole político estratégica, de más envergadura y como punta de proyección extrainstitucional e internacional. Estos dos niveles no se excluyen entre sí, pero deben funcionar a través de sus ámbitos y competencias bien definidas y delimitadas. Esta política institucional debe construir agendas estratégicas, que tracen las rutas temáticas de interés hacia el quinquenio y normen la producción académica. Puesto que en el Estado y su agenda nacional de investigación, el rubro seguridad es deficiente o inexistente, la ANSP tendrá la oportunidad de dotar al sector público de programas de investigación científica coherentes con las necesidades de seguridad del país e incluso aportando la línea de investigación nacional en la materia apuntada.

\section{Desafío estratégico \#2}

La ANSP deberá avanzar hacia la organización de un sistema de investigación institucional.Un diseño que es probable que trascienda un quinquenio, pero que es impostergable. Mediante este se constituiría la base normativa para el ambiente institucional necesario para la producción científica. No sólo sistematizaria un régimen meritocrático que daría fuerza a la cultura de la investigación; sino que volvería la generación de nuevo conocimiento un esfuerzo sostenido, orientado a la resolución de problemas y como componente consubstancial de la vida académica en el marco del concepto de una universidad de policia. 

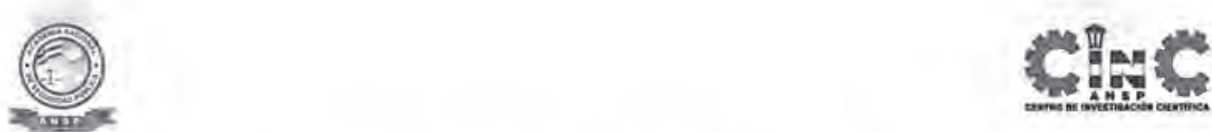

El sistema de investigación institucional deberá atender el sustento filosófico del marco educativo, definir politicas estratégicas claves concernientes a ética; que constituye la base para recompensar todo esfuerzo genuino del investigador o docente. Asimismo un área de producción intelectual y conocimiento en la que deben mediar incentivos morales, económicos y materiales para el académico hacedor de investigaciones; de igual manera implicará la vigencia y aceptación de un baremo que tome en cuenta aspectos fundamentales como formación profesional, publicaciones académicas por año, factores de impacto de las investigaciones entre otros asuntos relevantes. Otras áreas vitales del sistema de investigación corresponden a desarrollo cientifico y sector productivo, es decir cómo se construye la relación estratégica entre Estado, sociedad y economía; también la difusión del conocimiento, que implica cómo desplazarse de lo local a lo global y viceversa, determinándose con claridad los públicos estratégicos que se pretenden impactar e incidir. Las áreas de investigación / docencia y la administración financiera deberán plantear desde cómo la comunidad docente se apropia de formatos cientificos de trabajo y se incorpora a iniciativas académicas en desarrollo a otras que demandarán la inversión de determinados techos presupuestarios y porcentajes institucionales para el rubro investigacion cientifica.

\section{Desafio estratégico \# 3}

Las bases prioritarias para la institucionalización de la investigación científica en la ANSP, requieren la vigencia y autorización de algunos documentos estratégicos, principalmente cuatro. Uno que puede denominarse agenda de investigación en la que se describe un marco general para las temáticas estratégicas que atendiendo criterios de prioridad, oportunidad y factibilidad organicen los esfuerzos editoriales de las publicaciones científicas que se encuentren operativas. En segundo lugar otro que puede identificarse como Plan de Investigación y Análisis, que oriente, de vida y pensamiento estratégico a los programas de investigación cientifica en seguridad. Ambos existen pero sólo cubren el nivel de investigaciones político-estratégicas; la comunidad docente debe elaborar y diseñar documentos homólogos para la tarea de la investigación de tipo educativa-curricular.

El beneficio de los documentos en mención concierne a la posibilidad de convocatorias con antelación suficiente, proveen estabilidad y certidumbre para la escogitación de estudios periódicos y conceden una legitimidad de indole jerárquica pero funcional y práctica porque descomplica los tiempos prolongados de deliberación que dejan escasos resultados. Por ello la dependencia jerárquica de la unidad responsable de cada nivel y tipo de investigación es clave para un modelo exitoso. EI Centro de Investigación Científica debe seguir dependiendo de la Subdirección Ejecutiva y la Dirección General; mientras la investigación educativa acumulada de la comunidad docente deberá ser liderada por la Unidad 

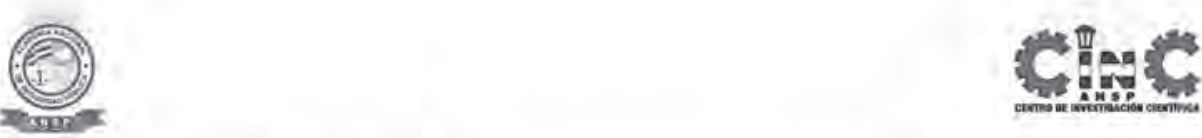

de Planeación Educativa (UPE); mientras sus documentos estratégicos deberán elevarse a la autorización de la Dirección General vía División de Estudios.

\section{Desafío estratégico \# 4}

Una revisión exhaustiva de documentos gubernamentales pertinentes, ha dejado como resultado el hecho que el Estado no cuenta con un programa de investigación científica en seguridad. Las alusiones al rubro son escasas, muy genéricas $y$ de limitada visión estratégica. Esa realidad complica el reconocimiento hacia la producción científica en el sector de la seguridad pública. Los especialistas e investigadores en seguridad no se encuentran inventariadosen las bases de datos de CONACYT y los documentos estratégicos que recogen las políticas nacionales de investigación, no cuentan a la seguridad ni como disciplina, ni como nicho en torno al cual puede y debe organizarse un programa de investigación científica; deshechándo por ende en cuanto asunto estratégico y de prioridad de país.

Lo anterior concede al Centro de Investigación Cientifica y por ende a la ANSP, la oportunidad de posicionar estratégicamente insumos fundamentales elaborados institucionalmente para la agenda nacional de seguridad y su componente investigación científica. Es lógico que puede adoptar una via de esa naturaleza, dado que los diseñadores y formuladores de políticas públicas, no siempre conocen las especificidades de los rubros y materias; a las que usualmente moldean para activarlos en instrumentos funcionales y operativos.

El interlocutor más calificado para generar lo antes expuesto es la misma comunidad de seguridad pública, un producto desde la estatalidad porque se pertenece a ella.

\section{Desafio estratégico \# 5}

El Centro de Investigación Cientifica debera construir y organizar un relacionamiento estratégico para los asocios internacionales; así como también los basados en el concepto de índole público-público.

Esto fortalecerá en primera instancia a nuestras publicaciones científicas, incorporará a otras entidades públicas cuyas especialidades sistémicas aportarán fundamentalmente al carácter transdisciplinario con la que debe dotarse a la investigación científica en seguridad y garantizará que nuestros esfuerzos locales trasciendan a un plano global. Asimismo propiciará sinergias sistemáticas entre los grupos estratégicos que conforman la comunidad de seguridad pública.

Lo anterior sólo puede operativizarse con instrumentos de convenios, cartas de entendimiento, formalización de diálogos extra institucionales e iniciativas y proyectos ad hoc para la investigación conjunta. Lo clave acá es prevenir la burocratización y la tentación jerárquica al sobredimensionaminto del proceso, sin que este no haya pasado por una etapa práctica previa y contribuido a la 

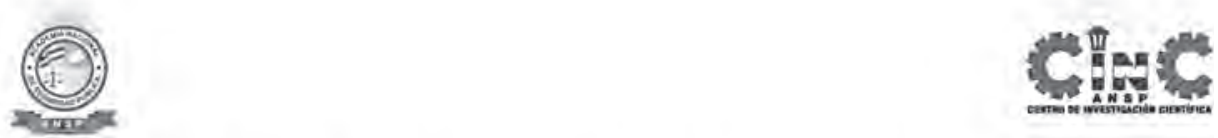

consolidación de resultados y gestión de posibilidades colaborativas pioneras y factibles.

\section{Desafio estratégico \#6}

Al término de la presente administración gubernamental se habrá consolidado la revista "Policía y Seguridad Pública" una publicación élite que deberá potencializarse. Ello requerirá de su acceso progresivo a bases de datos académicas internacionales, las indizaciones serán vitales tanto en cantidad como calidad; mientras el propósito fundamental se refiera entre otros a los siguientes aspectos: Primero, una mejor visibilización de nuestro journal científico, asequible para autores y pares evaluadores; como condicion indispensable para dar fuerza a los apoyos académicos diversos. En segundo lugar como plataformas globales de exposición de contenidos de nuestra producción nacional e institucional a la lectura y consulta de otras comunidades académicas. Y finalmente referenciar nuestro journal para los efectos de citaciones y estímulo a líneas de investigación pertinente en otras latitudes. De momento las indizaciones logradas incluyen Google Académico, el Sistema Regional en Línea de Revistas Cientificas de América Latina, el Caribe, España y Portugal (Latindex) y el LatinAmericaJournal Online (Lamjol) del Reino Unido. Se encuentra en proceso Dialnet, de la Universidad de la Rioja (España) y se llevará a cabo una política agresiva de inserción en otras bases como CLASE, HAPI, EBSCO, SCOPUS, DIGITALIA, REDALYC. El desafio de concretar estos ingresos y continuar completando parámetros de gestión editorial y de calidad científica; podrá contribuir para el largo camino de incorporar a nuestra publicación para la próxima década al ScienceCitationIndex, conocida como corriente principal de la ciencia que aglutina en su haber y clasificación solamente 8,000 revistas académicas élites muy rigurosas a nivel mundial de las cuales tienen presencia 16 revistas de América Latina, incluida una centroamericana del área de la biología y que en su naturaleza constituirá nuestra meta más ambiciosa. Esto último no será fácil porque deberá descansar en la expansión ordenada de la unidad, la idoneidad del personal y su debido financiamiento. Estas serán condiciones para desarrollar tareas complejas que plantearán desafios como el sostenimiento de la periodicidad, la inclusión de estudios con un significativo factor de impacto, la participación de académicos nacionales e internacionales reconocidos que endosen legitimidad a lapublicación, una expansión en los arbitrajes tanto en plazos para resolver como en número de participantes; diseño y observancia de formatos científicos que regulen procesos editoriales y de evaluacion; excelentes revisores de contenido y correctores de estilo, rigurosa prefesionalización de los menesteres de traducción de artículos y más aún incorporación de investigaciones y estudios traducidos en su totalidad al inglés, o surgidos de un arbitraje totalmente bilingüe. 

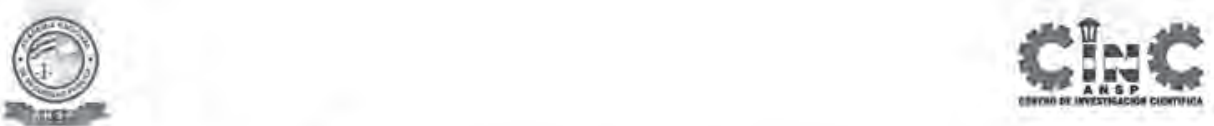

\section{Desafío estratégico \# 7}

Muchos de los asuntos descritos en este documento, no podrán realizarse, sí en el mediano y largo plazo, el Centro de Investigación Científica sigue contando con un equipo de trabajo basado en tres personas. Logicamente será necesaria la contratación de nuevas plazas permanentes e incluso dar pensamiento a otras modalidades que incluyan los medios tiempos (MT) o cuarto de tiempo (CT). El sostenimiento de la publicación "Policia y Seguridad Pública" tal y como está actualmente, es sencillamente insostenible para el largo plazo. Las razones que median en esa apreciación conciernen en otros asuntos con la saturación de procesos administrativos que elevan la carga burocrática y generan riesgos para el control de la calidad científica y editorial; así también las revistas arbitradas e indizadas llevan en forma inherente procesos internos de calidad y de obligatoria observancia, entre estas las fases de dictamen, etapas de cumplimiento de rutas de mejora, fases de verificación de rutas, correcciones de estilo, traducciones al inglés, revisión de al menos tres "domies" (prototipos) de imprenta; ello convive con la producción intelectual y esfuerzo propio de unidad para generar conocimiento nuevo, sin el cual las evaluaciones internacionales que se hacen sobre esos parámetros se verán deterioradas. Como coordinador principal del CINC, he aprendido y evaluado el desarrollo de esta experiencia y puedo dar fe que se ha hecho mucho con poco. De ausencia de presupuesto para la investigación, se ha evolucionado a la disponibilidad de determinados porcentajes. Los márgenes de no ejecución, en nuestro caso corresponden a los desajustes entre cíclos administrativos y ciclos científico-editoriales; es decir productos listos o de potencial preparación que se enfrentan a fechas críticas de recepción de requerimientos o incluso otras fechas límites para recepción de bienes y servicios. Asi estipula sus tiempos de gestión la administración pública, pero no significa que una institución educativa de nivel superior como la ANSP, no tenga claridad de los alcances de sus necesidades de investigación, con todo y la lógica, plazos y dinámicas con los que se devuelve; en todo caso, remover obstáculos institucionales conlleva a todo un desafio de modernización de lo público.

A la fecha he identificado al menos entre cuatro y cinco nuevas plazas necesarias para el desarrollo del Centro de Investigación Cientifica y su expansión de actividades inherentes al quehacer cientifico.

Dos plazas, definidas con perfiles de investigadores asistentes, vitales para apoyar los procesos editoriales claves; aumentar la producción de conocimiento elaborado en la institución e incursionar en la activación de otros formatosproducto de divulgación científica (anuarios, cuadernos, libros, ediciones especiales, etc.) Lo anterior incluye también el monitoreo a las etapas de filtro, revision y depuración en fases previas y posteriores al arbitraje. Una plaza más que incluya una persona con responsabilidad de gestión de proyectos de la 

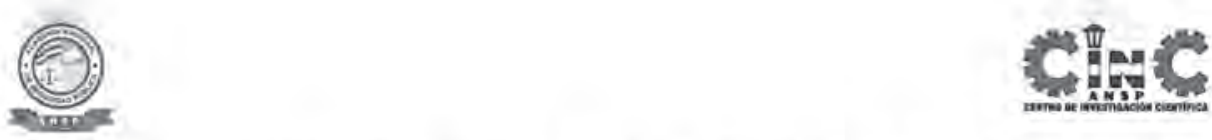

unidad para el ámbito de la cooperación internacional, pero la habilitación de un puesto así tiene su tiempo, no pueden iniciarse iniciativas de esta índole en forma masiva y luego carecer de planta de personal para procesar inteligentemente todos los compromisos. Tan solo la cooperación internacional para la investigación científica necesita su nicho y su cartera de proyectos. Los miembros restantes, uno deberá contar con especialidad informática y talentos para la administración de revistas virtuales que será la base de sostenimiento para las indizaciones. Manejar ambientes gráficos y trabajar con software de vanguardia, en cuanto herramientas avanzadas propiciadoras de plus y valor agregado en el sistema de consulta y oferta informativa en línea; naturalmente ya no circunscrito únicamente a la revista "Policía y Seguridad Pública" sino también cubriendo otras publicaciones o ediciones paralelas de las existentes y posiblemente en otras plataformas idiomáticas. Así también habilidades para trabajar con lenguajes de imprenta y simplificar los tiempos de las correcciones y diagramados.

Finalmente una persona para la gestión administrativa, un recurso humano clave para descomplicar la gestión diaria, con responsabilidades de sistematización de insumos de la unidad para cumplir requerimientos institucionales y tramitológicos ante el Departamento de Almacenes, monitoreo de solicitudes a la Unidad de Adquisiciones y Contrataciones Institucionales (UACI) ; diseño y elaboración de presupuestos anuales que se presentan a la Unidad Financiera Institucional (UFI); elaboración de informes mensuales y trimestrales requeridos por la Unidad de Planificación Institucional (UPI), evaluaciones periódicas entre otras actividades, si bien de rigor pero que generan interrupciones a los procesos fundamentales de la unidad; pero más grave aún impide que se desarrollen los ejercicios concretos de diseño, de ingeniería decisional, de elaboración de pensamiento estratégico; espacios y momentos necesarios para perseguir un futuro en materia de investigación cientifica. En la etapa para pensar la gran estrategia con la que se desean avanzar en los siguientes años.

La modalidad de reclutamiento que propongo por experiencia, resultados y por el imperativo de aseguranza de consistentes equipos de trabajo; se basa en el reclutamiento de jóvenes investigadores que puedan incorporarse a un empleo que funcione también bajo el concepto de escuela y fogueo; que estimule el talento para la investigación, que permita el aprendizaje sobre indizaciones, arbitrajes y demás procesos de la gestión cientifica editorial.

No es realista pensar en junior o sénior, que traen consigo formaciones superiores del extranjero, con expectativas salariales muy elevadas que no puede solventarlas el sector público por los desequilibrios de la estructura de incentivos económicos de la misma estatalidad. 

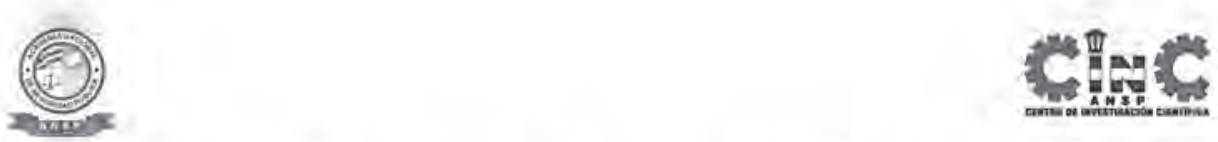

Además el riesgo de transmitir una cultura de prerrogativas anticipadas, sin tiempos especiales de demostración para generar resultados positivos y productos científicos, es un factor crucial de consideración a tener en cuenta. Dentro de un espacio de trabajo, son importantes desde aspectos como la inteligencia emocional, pasando por la gestión de la diversidad de talentos en una lógica convergente hacia la complementariedad y el clima laboral y de trabajo que se ofrece para que se materialice el desarrollo profesional; son muy importantes para observarse.

Los enfoques gerenciales modernos plantean al menos cuatro habilidades o talentos que deben estar distribuidos en todo equipo de trabajo porque es muy raro que estén todos presentes en una sola persona; y que de acuerdo a esta teoría somos una combinación de dos de los cuatro, pero que deben descubrirse. El grupo de los productivos, excelentes para resolver muchas tareas, ser efectivos en las mismas y perseguir la cuota de producción estipuladas según las plazas y asignaciones laborales. Los administradores que contribuyen al orden, a la sistematización de los documentos internos, su minucioso registro y periodización (estos son escasos); los emprendedores que sobre la base de la iniciativa, la creatividad y la innovación, diseñan las grandes cosas, visionan los impactos relevantes de sus ideas y los integradores, son las que procuran la cohesión interna del equipo de trabajo, el buen ánimo y garantizan una relación colaborativa permanente. Por ello es importante el proceso de seleccion, de buena gestión de entrevistas, esos atributos se advierten y se clasifican para organizar a equipos comprometidos con las metas. Finalmente dos cosas más en investigación y por la dinámica observada y aprendida en el seno del CINC, es importante el aspecto generacional, un equipo joven no está atado a esquemas preconcebidos; posee mayor margen para crear e innovar y abre brechas nuevas que impacten con cambios positivos en la estructura de la organización. El segundo aspecto, concierne a que influye mucho el origen del personal; mejor condición es el nuevo, el que viene de fuera. Tomar del interno (aunque en una primera etapa se haga por procedimiento); incrementa los riesgos de la contaminación, la predisposición, la transferencia de anticuerpos, el bloqueo interno y la imposición gradual de estilos obsoletos e ineficaces para hacer las cosas.

\section{Desafío estratégico \# 8}

Factor fundamental será para el corto y mediano plazo, la mejora salarial substancial para el actual equipo de trabajo del Centro de Investigación Científica. Esto responde a distintos aspectos que deben ser considerados. En primer lugar esta solicitud fue desestimada desde la etapa temprana de la vida de la unidad; la exigencia de estas condiciones no tiene cabida en un contexto en el cual el proyecto no ha generado resultados. Como conductor de la unidad hice conciencia desde el principio sobre la necesidad de trabajar duro, pero sobre todo 

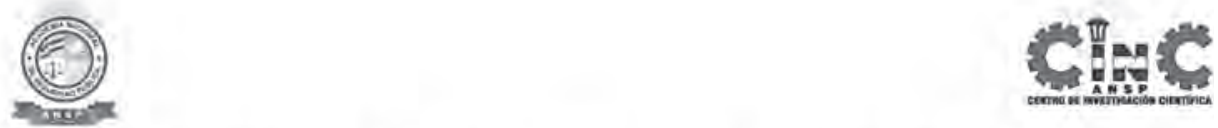

desarrollando una mistica conducente no solo al cumplimiento de metas, sino al logro de productos científicos de "excelente marca", implicando la calidad y diseño de mecanismos para dar vida a las politicas declaradas. Trabajando para la sobreproducción, cumpliendo algunas metas anuales en forma anticipada, generando jornadas especiales de trabajo más allá del horario normal; materializando apoyos amplios de colaboradores internos y pertenecientes a otras unidades de la ANSP, así también externos.

Lo anterior es la mejor carta de presentación para sustentar la solicitud referida al asunto salarial; este ritmo de trabajo que incluyó entre otros la organización del Centro de Información y Documentación (CEDIN); las indizaciones internacionales, los asocios estratégicos extra institucionales, el diseño web de un sitio CINC con URL propia y un concepto de revista virtual que aspiramos a desarrollar, lo anterior inevitablemente tiene un impacto y desgaste en los miembros del equipo, contrario a las ideas imperantes, no hay mejor inversion que hacerla en personal comprometido y que ha dado más de sí. La mejora salarial substancial concierne al mantenimiento de la moral del equipo, su estimulo inteligente y el reconocimiento a sus esfuerzos. Nuestro estilo ha sido trabajar duro por metas ambiciosas y al palpar resultados, comunicarlos; haciendo uso de la paciencia para esperar que la gestiones hechas fructificasen y perseverancia para no desistir de aquello que era posible, sin dejar de mencionar la disciplina como una constante para conquistar logros significativos y superiores.

Será de su conocimiento que los investigadores no son pagados como docentes, porque su misión es crear para resolver problemas, ayudar a pensar a dirigentes. y comunidad y ser pioneros en la exploración de todo aquel mundo que creemos descubierto. El concepto original de equipo materializador de apoyos, ha sido superado con creces; pues no resignándonos a fungir como gerentes administrativos, armadores y diseñadores; descubrimos en el proceso la misión imperativa de posicionar la marca de casa, sobre todo en un contexto de ausencia de cultura de investigación o elaboración de pininos de producción cientifica, básicamente incipiente que encontramos entre la comunidad docente.

Mi colaborador pronto debió abandonar la lógica de la cultura burocrática, y tomar la responsabilidad de incursionar en la producción escrita, en el sentido de hacer valer su formación universitaria. Pero más allá de eso, invirtió económicamente en formarse en gestión cientifica, también especializándose en el rubro, conociendo la gestión editorial y las tareas inherentes a la conducción de una unidad como el CINC; porque como personas no debe tenerse la competencia de los subalternos, sino ser sabios en hacerles crecer y tener incluso valentía y humildad para dar espacio. En todo este marco creamos de todo, hasta grupos de estudio adhoc con jornadas de trabajo semanal, 

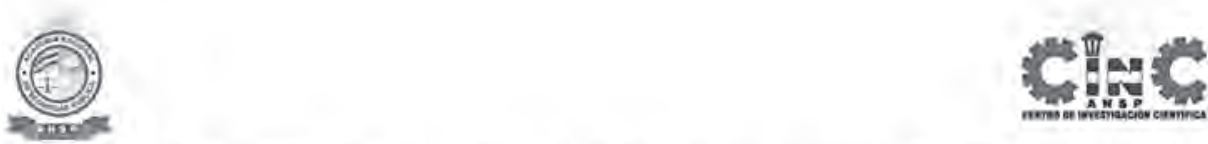

circuitos académicos en línea; pera más aún entusiasmando a otros en el Éstado, tocando puertas, exhortándoles e invitándoles; validando sus aportes.

En cuanto a ha cumplido más allá de su área de especialización en el rubro de la bibliotecología; ha tenido la habilidad de procesar inteligentemente las proyecciones que se plantean, cumplir creativamente las misiones y hacer más de lo necesario. Desde 2011 su apoyo para contactos académicos internacionales, búsqueda de árbitros, nichos potenciales para la cooperación entre otras han sido fundamentales. El acompanamiento al levantado de distintas secciones de la revista muy relevante, su papel en la coordinación con los colaboradores; pero el más importante su disponibilidad permanente y su capacidad de entusiasmar a otros sumando para los esfuerzos del CINC, sin dejar de mencionar además su invaluable apoyo a la investigación de los miembros del CINC, buscando información, sistematizando fuentes, apoyando en la digitación de manuscritos, corrigiendo versiones preliminares y de prueba.

Este servidor ha estado en todo lo anterior muy próximo, incluido y ha sido parte de todas estas iniciativas, haciendo el esfuerzo para que la melodía musical no fuese tocada a prisa, ni desafinada, ni lenta; sino generada en su tiempo y sabiamente.

\section{Desafio estratégico \# 9}

El Centro de Investigación Científica no puede resumirse en una publicación única, aunque sea pionera y vanguardista, Será imperativo avanzar a otros instrumentos divulgativos de conocimiento científico en seguridad, lo proyectado es mucho más amplio y progresivamente tomarán forma pero ello estará en función del crecimiento de planta de investigadores y otros apoyos, pues el concepto que buscará instalarse adoptará los sistemas de arbitraje, tendrá como objetivos la indización internacional y edificará fundamentos en los asocios estratégicos.

Entre estos productos, de los cuales se tienen diseños reticulares preliminares, encontrándose los siguientes:

1. Anuario de la Academia Nacional de Seguridad Pública, una edición especial que recogerá todos los estudios e investigaciones con temáticas misceláneas, que se ajusten a los formatos definidos en la revistas "Policía y Seguridad Pública"; que aprueben arbitrajes, cumplan rutas de mejora; pero que no correspondan al concepto monotemático de las convocatorias semestrales. 

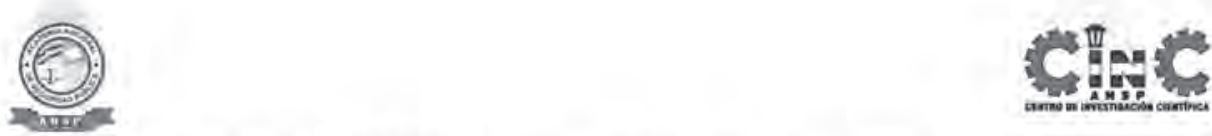

2. Cuadernos de la ANSP, el instrumento ideal para recoger y sistematizar la investigación educativa, curricular e institucional. Una publicación apoyada en un sistema de arbitraje en dónde participe y conduzca activamente la comunidad docente. Esta deberá atender el diseño de un programa de investigación y una agenda estratégica aprobados por el Director General. Esta colección que deberá tener una periodicidad semestral, apoyándose en la producción académica docente y en el marco de que se habría avanzado en la implementación de una política de investigación y un sistema de investigación institucional. Sistematizará los mejores estudios elaborados por los policias, preferentemente de niveles intermedios en adelante y se abrirá a otros aportes nacionales e internacionales de instituciones homólogas, al menos de la región centroamericana.

3. Cuadernos de investigación en seguridad, que observará los parámetros internacionales de la gestión científica y editorial, atenderá temáticas estratégicas emergentes o acordados en el marco de la activación de programas de investigación científica del Centro de Investigación Científica. Podrá iniciarse con un volumen por año y luego evolucionar a dos. Y podrá tratar temas prioritarios coyunturales que se moldeen con mayor flexibilidad que el régimen monotemático semestral.

Pero el desarrollo de publicaciones científicas, relevantes e indizadas no pueden convertirse en un fin en si mismo. Más importante será la evolución a mayores propuestas de inversion para la investigación cientifica, incluso llegándolo a fijar por ley en un porcentaje substancial respecto a los totales asignados por ano. Esta inversión debe plantear en forma creciente y decisiva la investigación más desarrollo (I+D); que sea capaz de propiciar incluso patentes, métodos, proyectos avanzados, productos vanguardistas de implicación práctica, diseños transdisciplinarios.

A manera de ejemplo me permito ilustrar con tres esbozos de proyectos estratégicos que en algún momento el Estado deberá impulsar y diseñar inteligentemente:

1. Desarrollo de software de simulación para entrenamiento y planificación avanzada para cuadros policiales en la gestión y manejo de situaciones críticas tales como: toma de rehenes, sabotaje, actos terroristas, desbordamientos de protestas públicas, alaque a infraestructuras estratégicas, entre otras. Para ello deberá migrarse decenas de planes interiores de edificios públicos a formatos virtuales, ello puede contribuir a la disponibilidad de herramientas claves para el planeo de operaciones, la organización de dispositivos de seguridad, entre otros asuntos 

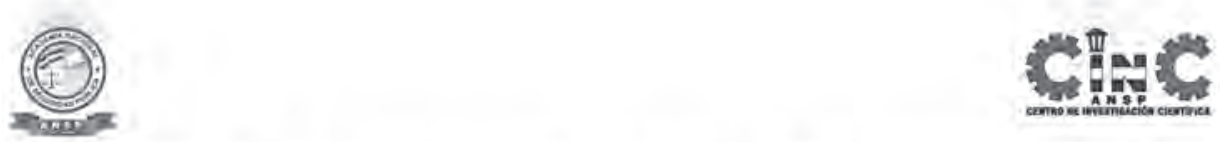

fundamentales porque se proporcionarian de dimensiones espaciales, diagnósticos de infraestructura, vulnerabilidades potencialidades para su protección.

2. Los servicios esenciales que la ANSP recibe anualmente y que se traducen en montos especificos significativos en facturas de electricidad, que es vital para la vida y funcionamiento de una institución pública, por todos los procesos inherentes en su gestión, desarrollo y cumplimientos de metas. Puede ser progresivamente sustituido por una transición ordenada y planificada a las energias renovables. Con una inversión estratégica anual para la investigación más desarrollo $(I+D)$, de la ANSP podría iniciar desde los estudios de factibilidad, pasando por planes con sus respectivas etapas, hasta la instalación decisiva de dispositivos tecnológicos tipo paneles solares; o reciclamiento de basura o generación de biogás procesando las excretas humanas para los efectos de extracción de metano. Una ruta para la independencia energética de una institución pública podria significar, perseverar por al menos 8 años y podría generar un efecto imitativo en el resto del Estado. Recuèrdese que las energías limpias renovables son un programa prioritario en la agenda de investigación nacional actual.

3. El mapeo o levantamiento estratégico biopsico social y clínico de todos los miembros de la corporación policial. Este inventario especializado permitiria conocer cuántos policías tienen lesiones incapacitantes producto del cumplimiento del deber, que impacto tiene eso en la reubicación de los mismos en otros puestos y en el rendimiento general de la organización y sus dinámicas salariales. Permitiria la cuantificación de eventos de estrés post traumático a los que se encuentran expuestos en la cotidianidad de su labor y en contextos de violencia elevada, inseguridad compleja y deficientes condiciones de clima de trabajo y régimen laboral.

Los propósitos de este documento estratégico ha sido el de pensar el futuro, para una unidad prototipo cuya visión puede llegar tan lejos como se fijen sus umbrales y por ello todo su quehacer intelectual deberá sujetarse a las siguientes premisas:

a) Pensamiento estratégico, para saber qué hacer y cómo hacer. Se trata del sustento vital que da forma a las grandes iniciativas, asume responsabilidades y visiona estados de salida asequibles, en concreto imagina los largos plazos.

b) Pensamiento creativo, para generar propuestas nuevas, ingeniosas y realmente movilizadora de capacidades para imaginar soluciones, implementar formas prácticas y lidiar flexiblemente con lo complejo. 

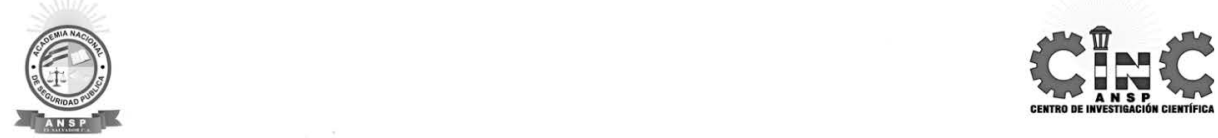

c) Pensamiento innovador, para generar procedimientos, políticas y estrategias; relevante para conducir hacia la administración inteligente de valores agregados de estados sucesivos de plusvalía para los grandes conceptos e ideas fuerza.

d) Pensamiento avanzado, para impactar con esfuerzos pioneros que abren brechas nuevas, que vuelven vanguardistas a las iniciativas y que hacen que instituciones, personas y liderazgos se adelanten a sus tiempos.

e) Pensamiento prospectivo, para imaginar los futuros deseables y adversos; propiciar sinergias y tratamientos en tiempo presente, asumiéndolos en sentido de alta probabilidad pero para adecuarse a la gestión de los retos.

f) Gestión del talento humano, para identificar apoyos, personas claves, reubicar sus talentos y habilidades y generar una capacidad significativa de rendimiento y compromiso.

Todo lo anterior con el conocimiento (saber acumulado, oportuno y apropiado); la inteligencia (para advertir consecuencias); el discernimiento (para diferenciar rutas fáciles, equivocadas, inoportunas o ruinosas); la prudencia (para saber cuándo hacer un alto, plantearse alternativas o aceptar variantes de las rutas originalmente concebidas y finalmente con sabiduría (para aprender de todos, extraer lo mejor de los otros incluyendo a nuestros críticos, amigos y enemigos). Con la disposición de aprender, y extraer y procesar lecciones de la escuela de la vida misma. Esta es una base para una filosofía de trabajo muy superior a la cultura de escasez y miseria intelectual que actualmente invade a la civilización contemporánea en todos los frentes.

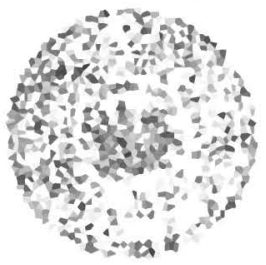


